\title{
Estudio morfológico del oído medio e interno de la Chinchilla laniger*
}

\section{Morphological study of the middle and internal ear of Chinchilla laniger}

\author{
Loreto Carrasco $\mathrm{M}^{1}$, J. Cristóbal Maass $0^{1-3}$, Luis Dentone $\mathrm{S}^{2}$, Gonzalo Miranda $\mathrm{G}^{4}$, Manuel Kukuljan P1.
}

\begin{abstract}
RESUMEN
Introduccón: La Chinchilla laniger, roedor originario de la Cordillera de Los Andes, ha sido utilizada como modelo de investigación otológica por más de 40 años. Sin embargo, no existe un estudio anatómico e histológico detallado del oído de este animal. La mayoría de las investigaciones se han desarrollado en neurofisiología de la audición.

Objetivos: Describir macroscópica y microscópicamente el oído medio e interno de la Chinchilla laniger.

Material y método: Estudio realizado en cinco chinchillas adultas (previa aprobación del Comité de Bioética sobre la Investigación en Animales). Macroscopía: Se realizó una cirugía vía conducto auditivo externo y se estudió también con una tomografía computada de oídos. Microscopía: Disección, fijación e inclusión en parafina. Tinción con hematoxilina-eosina

Resultados: Vía conducto auditivo externo se realiza un colgajo timpanomeatal similar al de estapedectomía Se accede a la caja timpánica por el cuadrante posterosuperior. Se observa que el martillo presenta un delgado mango unido verticalmente al tímpano. Presenta una cabeza con un gran proceso anterior en vez de ligamento timpanomaleolar anterior. Su cabeza se une al yunque, fusionándose. $\theta$ proceso lenticular del yunque se une a un delgado estribo, en el cual se observa el vestigio de la arteria estapedial, entre las cruras. $\theta$ promontorio es visible fácilmente dado su tamaño y prominencia La ventana oval se encuentra levemente más superoanterior que la ventana redonda. La cóclea da 3 y media vueltas sobre el modiolo. 日 órgano de Corti, sáculo, utrículo y crista ampularis se identificaron en los cortes histológicos.

Conclusiones: $\theta$ estudio morfológico detallado de un modelo de investigación como la chinchilla es imprescindible para el desarrollo de nuevos estudios en audición.

Palabras clave: Chinchilla laniger, oído medio, oído interno.
\end{abstract}

\footnotetext{
Médico, Laboratorio de Neurobiología Celular y Molecular, ICBM, Facultad de Medicina Universidad de Chile.

Médico, Servicio de Otorrinolaringología Hospital Clínico Universidad de Chile

Médico, Servicio de Otorrinolaringología Clínica Alemana de Santiago

Médico, Servicio de Radiología Hospital Clínico Universidad de Chile
} 


\section{ABSTRACT}

Introduction: Chinchilla laniger is a rodent that has been used as a model of ear research for more than 40 years; nevertheless, a macroscopic and microscopic detailed study of the ear in this animal is lacking.

Purposes: To describe the anatomy and histology of the middle and inner ear of Chinchilla laniger.

Materials and methods: Five adult chinchillas were used in this study. The macroscopic study was made with a computed tomography of the ear and then dissection under a microscope. For the microscopic study, ears were dissected, fixed, decalcified, included in paraffin and then sectioned and stained with hematoxylin-eosin.

Results: A tympanomeatal flap was made via the external acoustic meatus, similar to a stapedectomy. The tympanic cavity was accessed through the posterosuperior quadrant to examine the malleus. A thin manubrium attached vertically to the eardrum could be observed. The malleus head is characterized by a large anterior process, instead of the anterior tympanomallear ligament, and it was fused to the incus. The lenticular process of the latter joined a thin stapedius, in which the vestige of the stapedial artery was observed (between the cruras). The promontory was visible easily because of its size and prominence. The oval window was located more superoanterior than the round window. The cochlea gave 31/2 turns around the modiolus. The organ of Corti, saccule, utricle and ampullary crest were identified in the histological sections.

Conclusions: The ear of Chinchilla laniger is similar to the human ear, supporting its use as a model in ear research. The study of the ear, specially the cochlea, is difficult and needs great dedication in order to obtain good results.

Key words: Chinchilla laniger, middle ear, inner ear.

\section{INTRODUCCIÓN}

La chinchilla es un roedor originario de la Cordillera de Los Andes, perteneciente a la familia Chinchillidae. El género chinchilla comprende 2 especies: C. lanígera (Chinchilla laniger) y la C. chinchilla (Eyomis brevicaudata) ${ }^{1,2}$.

La crianza de chinchilla se ha difundido a nivel mundial por varias razones, ya sea para producir mascotas, reproductores, pieles y también para la producción de animales de experimentación.

La chinchilla comenzó a ser utilizada como modelo de investigación otológico por Rasmussen y posteriormente por Miller en el año $1963{ }^{(3,4)}$. El interés por este roedor fue en aumento por varios motivos: 1) es un animal fácil de mantener en un laboratorio siendo lo suficientemente grande para soportar cirugías otológicas, 2) presenta baja incidencia de infecciones espontáneas del oído medio, 3) su vida es relativamente larga, de 15 a 20 años, por lo que el factor tiempo no es un problema en estudios a largo plazo, 4) puede ser entrenada para realizar estudios de tareas auditivas, 5) su rango de audición y sensibilidad auditiva es similar a la del humano, 6) la presencia de su gran bula permite una firme fijación de la cabeza y un acceso quirúrgico directo a través de ésta ${ }^{5,6}$.

A pesar de que la chinchilla se ha utilizado por más de 40 años en investigación otológica, no existe un estudio anatómico e histológico detallado del oído de este animal, ya que la mayoría de las investigaciones se desarrollan en el área de la neurofisiología de la audición.

Una característica especial del hueso temporal de la chinchilla, es la presencia de bulas (una a cada lado), que corresponden a grandes cavidades aireadas delimitadas por tejido óseo fino y que en su interior presentan un número variable de tabiques. Se ha subdividido la bula en una parte dorsal, mastoidea y laberíntica. La parte dorsal es subcutánea, y por lo tanto, palpable. La parte mastoidea de la bula está separada internamente por un septo, y no puede ser palpada externamente ya que está cubierta por el músculo occipital. La 
parte laberíntica, a pesar de estar cubierta por el músculo digástrico, es palpable en la región cervical. Toda esta región es ventilada a través de la tuba auditiva?.

Por último, el oído interno de este animal, específicamente el laberinto membranoso anterior (coclear), presenta la forma de un conducto que da tres y media vueltas en relación a una estructura central o modiolo?.

En resumen, debido a la amplia difusión de la chinchilla como modelo de investigación en otología, es necesario estandarizar un método para el estudio morfológico del oído de ésta, describiendo tanto las técnicas utilizadas como la metodología para que posteriormente se logren reproducir. Además, la descripción detallada de la anatomía macroscópica y microscópica con registros de fotografía digital es fundamental para confeccionar un atlas de la anatomía del oído de la chinchilla, el cual será de gran utilidad para los investigadores que requieran abordar quirúrgicamente el oído 0 estudiar su histología. Por último, resulta necesario adquirir la destreza de estudiar histológicamente el órgano coclear, ya que en nuestro país existe poca experiencia al respecto, lo cual limita a los clínicos e investigadores al estudio histopatológico de enfermedades relacionadas con este órgano.

El objetivo de este trabajo consiste en describir la anatomía y la histología del oído medio e interno de la Chinchilla laniger y de esta forma obtener un atlas de anatomía e histología del oído de este animal que sea de fácil acceso.

\section{MATERIAL Y MÉTODO}

\section{Animales}

Las chinchillas se obtuvieron de un criadero autorizado para el comercio peletero. Los procedimientos llevados a cabo fueron evaluados y aceptados por el Comité de Bioética sobre la Investigación en Animales, de la Facultad de Medicina, Universidad de Chile (CBA \# 0240 FMUCH).

\section{Descripción macroscópica del oído medio e interno}

Este procedimiento se realizó en 1 Chinchilla laniger adulta sana. Bajo sedación se sometió a una tomografía computada (TC) de oídos, en el Servicio de Radiología del Hospital Clínico de la Universidad de Chile (TC multicorte de 40 canales Siemens) Se realizaron cortes de $0,6 \mathrm{~mm}$ con reconstrucciones cada $3 \mathrm{~mm}$. Para la sedación se utilizó xylazina (2 mg/kg), atropina $(0,04 \mathrm{mg} / \mathrm{kg})$ y ketamina $(10 \mathrm{mg} / \mathrm{kg})$ intramuscular.

Una vez realizada la evaluación del examen imagenológico, se continuó con la segunda parte del estudio, que consistió en abordar quirúrgicamente el oído de la misma chinchilla, obteniendo un registro fotográfico de la anatomía. Se anestesió con atropina $0,04 \mathrm{mg} / \mathrm{kg}$, xylazina 3 $\mathrm{mg} / \mathrm{kg}$ y ketamina $40 \mathrm{mg} / \mathrm{kg}$ intramuscular, con refuerzos con ketamina $10 \mathrm{mg} / \mathrm{kg}$ cada 30 a 45 minutos 0 antes si era necesario, evaluando la respuesta a la presión de la pata posterior que se realiza suavemente con una pinza (lo que determina si el animal está o no bajo anestesia profunda).

La cirugía se realizó en una sala adaptada para este fin. Se realizó una incisión periauricular, separando las partes blandas del tejido óseo. Bajo microscopio (Zeiss, OPMI 1) se confeccionó un colgajo timpanomeatal para acceder al oído medio. Se identificaron las estructuras del oído medio e interno.

Se obtuvieron registros con fotografía digital (Digilife $^{\circledR}$ modelo DDV-M1 de 11 megapixeles) de cada estructura identificada.

La eutanasia se realizó con la chinchilla anestesiada profundamente, inyectando tiopental sódico $120 \mathrm{mg} / \mathrm{kg}$ (intracardíaca).

\section{Descripción del procedimiento histológico}

Se utilizaron 4 Chinchillas laniger adultas sanas (8 oídos).

Tras someterlas a eutanasia (método descrito en la sección anterior) cada cabeza se fijó en formalina al $10 \%$ (fijador universal) o paraformaldehído al $4 \%$. Se fijó mínimo por 24 horas para luego descalcificarla.

Por lo tanto, se retiró de la solución fijadora tras 24 horas en ella, se lavó con agua corriente e inmediatamente se dejó en $100 \mathrm{ml}$ de ácido tricloroacético al 2,5\% por aproximadamente 2 semanas, realizando un cambio de la solución tres veces a la semana (lunes, miércoles y viernes) y manteniéndola a temperatura ambiente. Para eva- 
luar la descalcificación, se utilizaron pruebas radiológicas o métodos químicos. Para realizar la inclusión en parafina del tejido en estudio, éste fue sometido a un proceso de deshidratación y luego impregnación en parafina.

Una vez obtenido el taco de parafina, ya solidificado, se realizaron los cortes en un micrótomo de rotación (Jung). Se utilizaron navajas para tejido duro 0 semiduro (Leica 819 de perfil bajo). Se realizaron cortes de 5 a 10 micrómetros de grosor. Los cortes histológicos de oído que se obtuvieron se montaron en portaobjetos tratados previamente con una solución adherente (gelatinizados 0 albuminizados).

Los cortes histológicos se realizaron con orientación horizontal para facilitar posteriores comparaciones.

A continuación, se realizó tinción corriente con hematoxilina-eosina. La hematoxilina da la tinción nuclear y la eosina permite dar contraste, tiñendo de rosado el citoplasma de las células y de rojo brillante el colágeno y el tejido muscular.

Finalmente, una vez obtenida la placa histológica, se describe el oído del animal.

\section{RESULTADOS (Tabla 1)}

\section{Membrana timpánica}

Al igual que en el humano la superficie de la membrana timpánica se divide en pars tensay pars fláccida La primera, corresponde a la mayor parte del tímpano, mientras que la pars fláccida está formada por la pequeña porción de tímpano superior limitada por los ligamentos del martillo a nivel del proceso lateral de éste osículo y el borde superior de la membrana (Figura 1). El diámetro mayor fue de $8,5 \mathrm{~mm}$, con un área de $55,7 \mathrm{~mm}^{2}$ aproximadamente.

\section{Oído medio}

Se realizó un abordaje timpanomeatal de 12 a 6 del oído izquierdo de la chinchilla estudiada, similar a la de estapedectomía, accediendo fácilmente al oído medio izquierdo de la chinchilla. Se accede a la caja timpánica por el cuadrante posterosuperior observando así su contenido.

El martillo tiene dos partes principales: la cabeza, que articula con el yunque en la articulación incudomaleolar; y el mango o manubrio, que se adhiere a la membrana timpánica. Además, presenta dos apófisis o procesos: uno anterior (el cual es más grande), y uno lateral más pequeño que también se une al tímpano. La región entre el mango y la cabeza se denomina cuello (Figura 2).

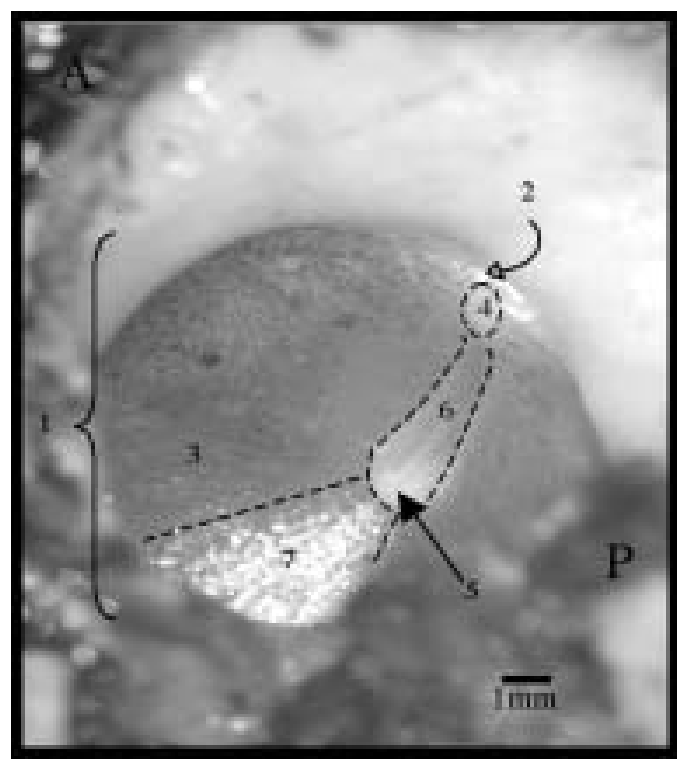

Figura 1. Cara lateral de membrana timpánica oído izquierdo de chinchilla. 1. Membrana timpánica; 2. Pars fláccida: 3. Pars tensa; 4. Apófisis corta del martillo; 5. Umbo; 6. Mango del martillo; 7. Triángulo luminoso. A. anterior; P. posterior.

Tabla 1. Resumen de chinchillas estudiadas

$$
\begin{aligned}
& 1 \text { Chinchilla laniger Tomografía computada y estudio macroscópico (cirugía) } \\
& 4 \text { Chinchilla laniger Histología }
\end{aligned}
$$


El yunque se puede dividir en tres partes principales: un cuerpo y dos procesos (largo y corto) (Figura 3). El cuerpo articula con la cabeza del martillo, y en este animal se encuentran parcialmente fusionados (Figuras 4 y 5). El proceso largo se encuentra en una posición vertical, y en su extremo inferior se encuentra una región denominada proceso lenticular, el cual articula con el estribo. El proceso corto del yunque se orienta más

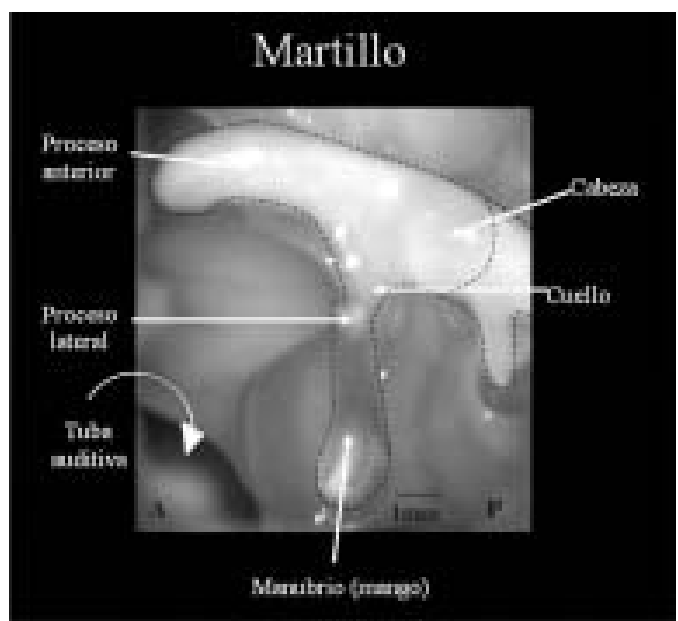

Figura 2. Martillo oído izquierdo de chinchilla (in situ). Cara lateral del martillo. Hacia anterior se observa el comienzo de la tuba auditiva. A: anterior; P: Posterior. horizontal y se une a la pared posterior de la cavidad timpánica a través del ligamento posterior (ligamento ancho) del yunque.

La longitud de esta barra ósea que se forma al estar ambos osículos fusionados mide aproximadamente $7,8 \mathrm{~mm}$. El mango tiene una longitud 4,3 $\mathrm{mm}$ mientras que el proceso largo del yunque mide $1,6 \mathrm{~mm}$ y el corto $0,7 \mathrm{~mm}$ aproximadamente.

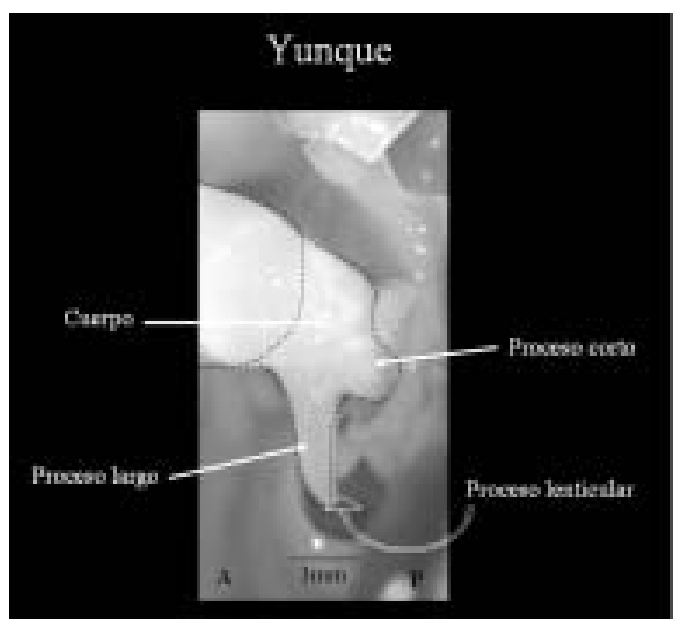

Figura 3. Yunque de oído izquierdo de chinchilla (in situ). Se pueden observar sus distintas partes: cuerpo, proceso corto, largo y lenticular (este último articulando con el estribo). A: anterior; P: posterior.

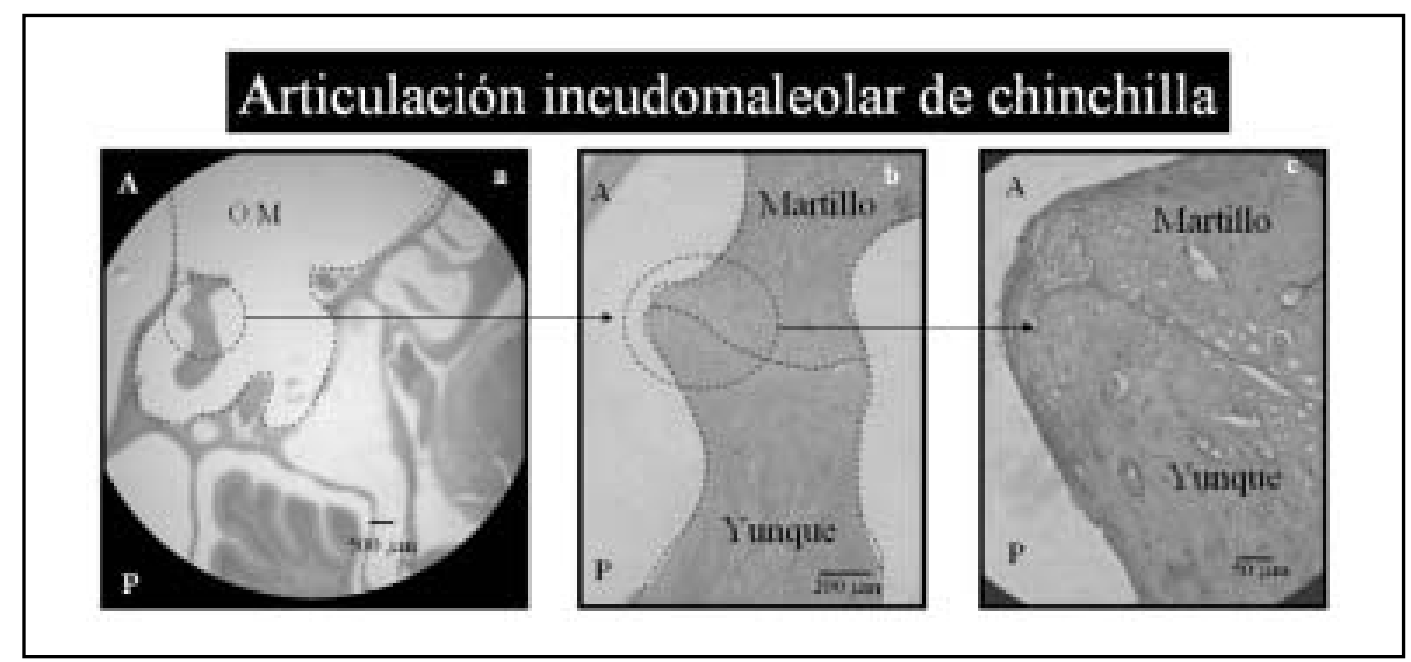

Figura 4. Microscopía de la articulación incudomaleolar de chinchilla. En a se observa parte del oído medio (0.M), y en él parte de la cadena osicular, específicamente la articulación incudomaleolar. En b y c se observa ésta con un mayor aumento, destacando que está casi completamente fusionada. A. Anterior; P. Posterior. 


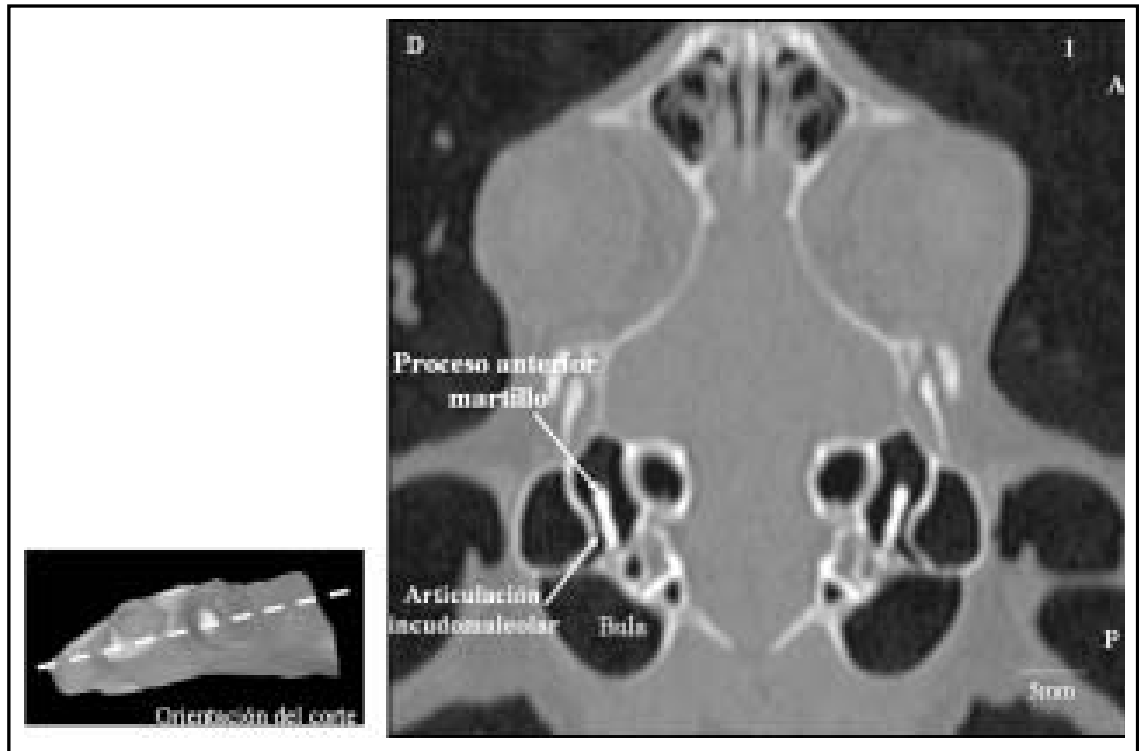

Figura 5. Imagen de TC de chinchilla, corte horizontal, en el cual se observa parte de la cadena osicular (martillo y yunque) y las bulas. A: anterior; P: posterior. D: derecha; I: izquierda.

El estribo está formado por cuatro partes: la cabeza, dos pilares o cruras (anterior y posterior) y la base o platina (Figura 6). La cabeza articula con el proceso lenticular del yunque en la articulación incudoestapedial. La platina cubre la ventana oval y tiene un área aproximada de $2,1 \mathrm{~mm}^{2}$. La distancia entre la base del estribo y la articulación incudoestapedial es de $1,7 \mathrm{~mm}$.
El diámetro mayor de la ventana oval fue de 2,23 mm y $0,73 \mathrm{~mm}$ como diámetro menor (con área de $1,8 \mathrm{~mm}^{2}$ ). Y por otra parte, la ventana redonda tiene un diámetro de $1,11 \mathrm{~mm}$ (con área aproximada de 1,29 $\mathrm{mm}^{2}$ ).

Esto determina una relación entre la superficie de la ventana oval y membrana timpánica de aproximadamente 1 es a 30 .

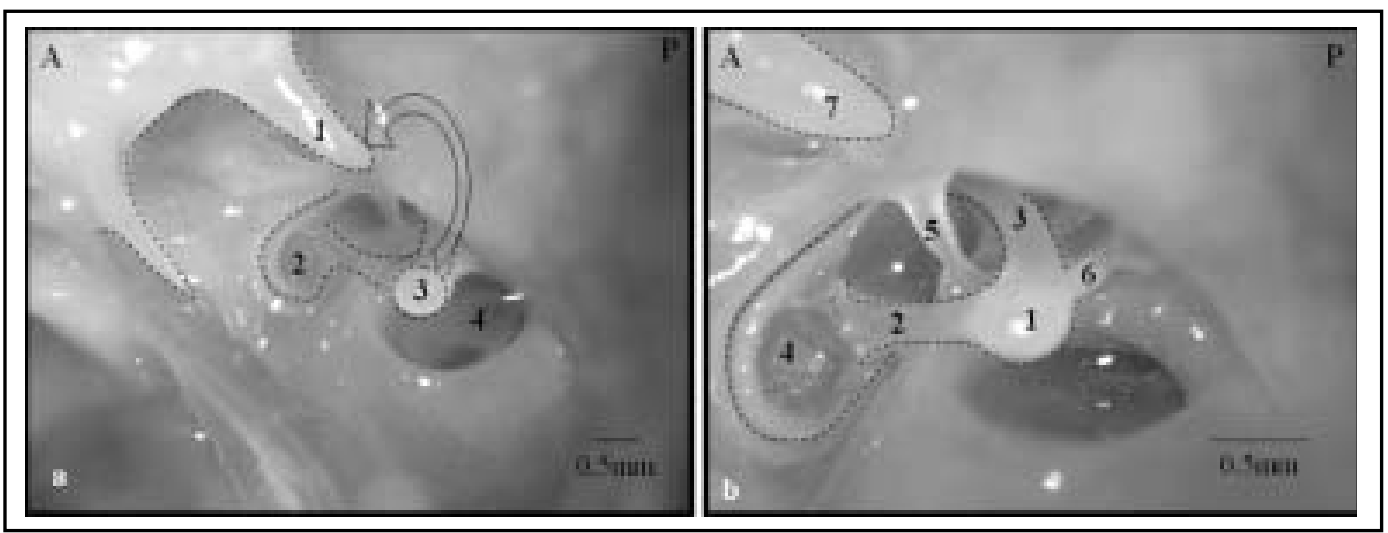

Figura 6. Estribo de chinchilla in situ. A: anterior; P: posterior. a. Desarticulación incudoestapedial (flecha) con el fin de observar el estribo. 1. Proceso largo del yunque; 2. Platina del estribo cubriendo la ventana oval; 3 .Cabeza del estribo; 4. Ventana redonda. b. Estribo in situ. 1. Cabeza del estribo; 2. Crura anterior; 3. Crura posterior; 4. Platina estribo; 5. Vestigio arteria estapedial; 6. Músculo del estribo. 7. Proceso lenticular del yunque. 


\section{Oído interno}

Al igual que en el humano, el oído interno está compuesto por un laberinto óseo y un laberinto membranoso. El laberinto óseo está formado por tres partes: la cóclea hacia anterior, el vestíbulo al centro y los conductos semicirculares hacia posterior (Figura 7). La cóclea está compuesta por un tubo enrollado alrededor de un eje central denominado modiolo, dando tres y media vueltas en esta especie (Figura 8). En el interior del modiolo se encuentra el ganglio espiral. Este tubo está dividido en toda su longitud por un tabique óseo y membranoso en tres escalas: vestibular, media y timpánica. La escala media, parte del laberinto membranoso, contiene el órgano coclear 0 de Corti (Figura 9).

El órgano de Corti se encuentra sobre la mem- brana basilar y bajo la membrana tectoria. Está compuesto por un grupo de células de sostén, una fila de células ciliadas internas y tres filas de células ciliadas externas. Las células pilares, externas e internas, se oponen entre sí formando un canal triangular, el túnel de Corti (Figura 10)

Los conductos semicirculares se ubican posterosuperior al vestíbulo. Son tres: el anterior, posterior y lateral. Todos nacen del vestíbulo, a partir de dilataciones denominadas ampollas, donde se encuentran terminaciones nerviosas del nervio vestibular (Figura 11). El conducto semicircular anterior y el posterior se unen en la crus común, antes de su desembocadura en el vestíbulo, formando la rama común, mientras que el conducto semicircular lateral desemboca en forma aislada (Figura 12).

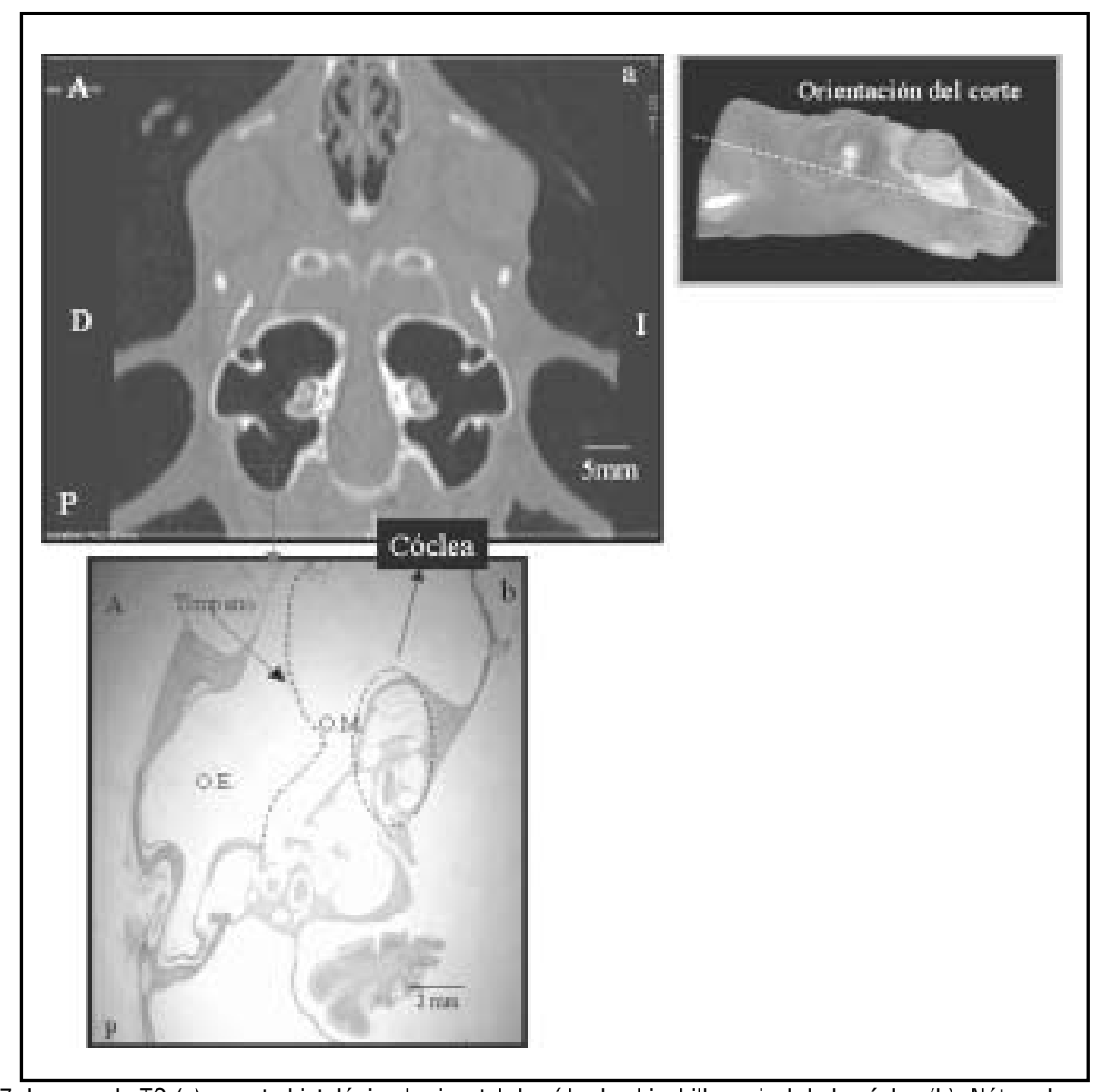

Figura 7. Imagen de TC (a) y corte histológico horizontal de oído de chinchilla a nivel de la cóclea (b). Nótese la proximidad entre la cóclea y el tímpano. 0.E.: oído externo; 0.M: oído medio. A. Anterior; P. Posterior; D: derecha; I: izquierda. 


\section{DISCUSIÓN}

En este trabajo se puede reafirmar que hay diferencias y semejanzas entre la chinchilla y el humano. Algunas de estas diferencias son favorables para el acceso quirúrgico, como por ejemplo, la presencia de bulas. Sin embargo, dado que el meato acústico de la chinchilla viaja de dorsal a ventral dentro del hueso temporal (quedando su pared lateral en el mismo plano de la pared de la bula laberíntica y mastoídea ${ }^{7}$ ) hace más difícil un acceso por esta vía. Otras características importantes de discutir son las siguientes:

a. Tímpano y oído medio

La membrana timpánica de la chinchilla, al igual que en el cobayo ${ }^{8}$, es dividida en forma simétrica por el mango del martillo (extremadamente fino), el cual se dispone vertical y central en el tímpano. En la chinchilla, este osículo divide a la membrana en dos áreas similares (una anterior y otra posterior); en cambio, en el humano y en el gato, esta división es asimétrica (el área posterior es mayor $)^{8}$.

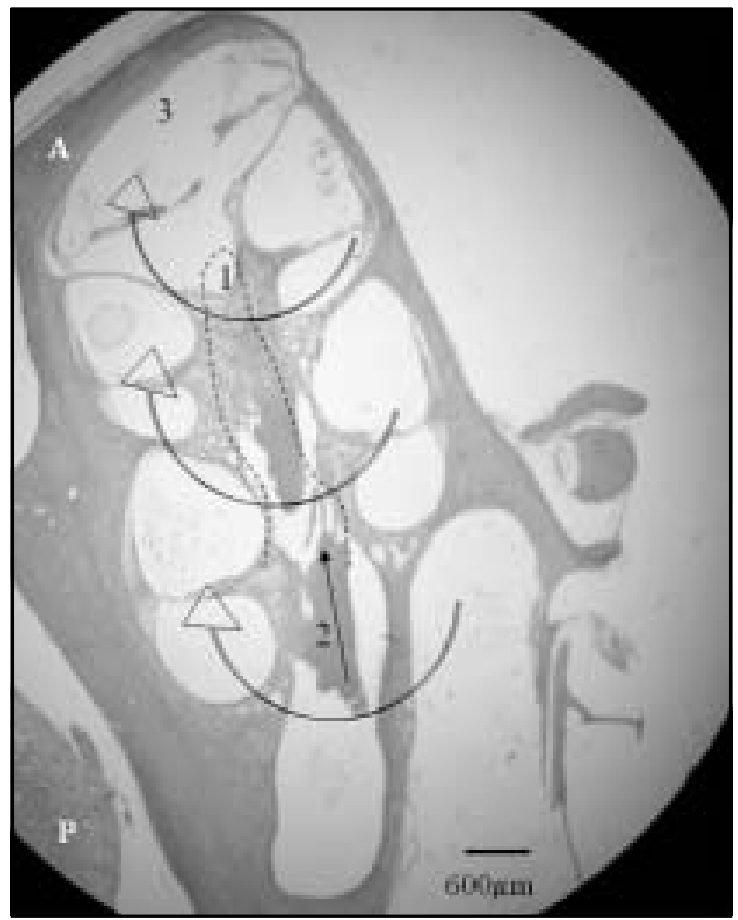

Figura 8. Corte histológico de cóclea de chinchilla a través del modiolo. 1. Modiolo; 2. Nervio espiral; 3. Ápex coclear. A. Anterior; P. Posterior. Flechas curvas indican vueltas de cóclea sobre modiolo.

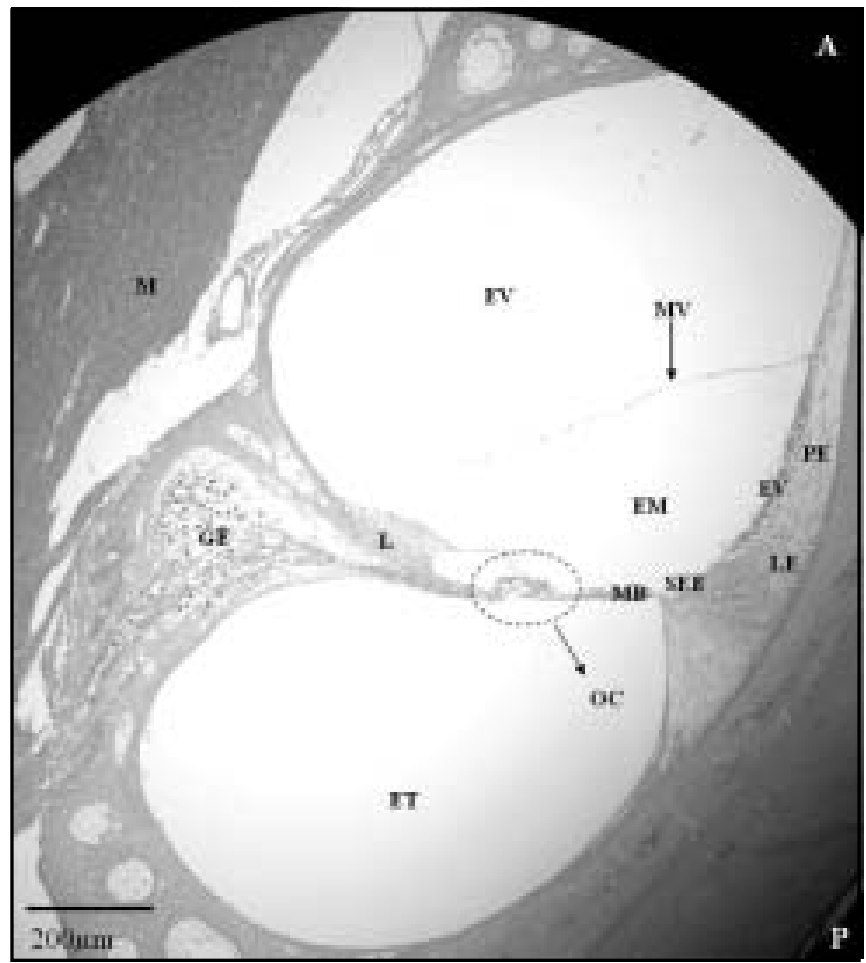

Figura 9. Aproximación a una espira de la cóclea de chinchilla. MV. membrana vestibular (de Reissner); EV. Escala vestibular; EM. Escala media; ET. Escala timpánica; MB. Membrana basilar; M. modiolo conteniendo fibras nerviosas; PE: pared externa (pared lateral); L. limbo espiral; OC. Órgano de Corti; GE. Ganglio espiral de la cóclea; EV. Estría vascular; LE. Ligamento espiral; SEE. Surco espiral externo. A. Anterior; P. Posterior. 


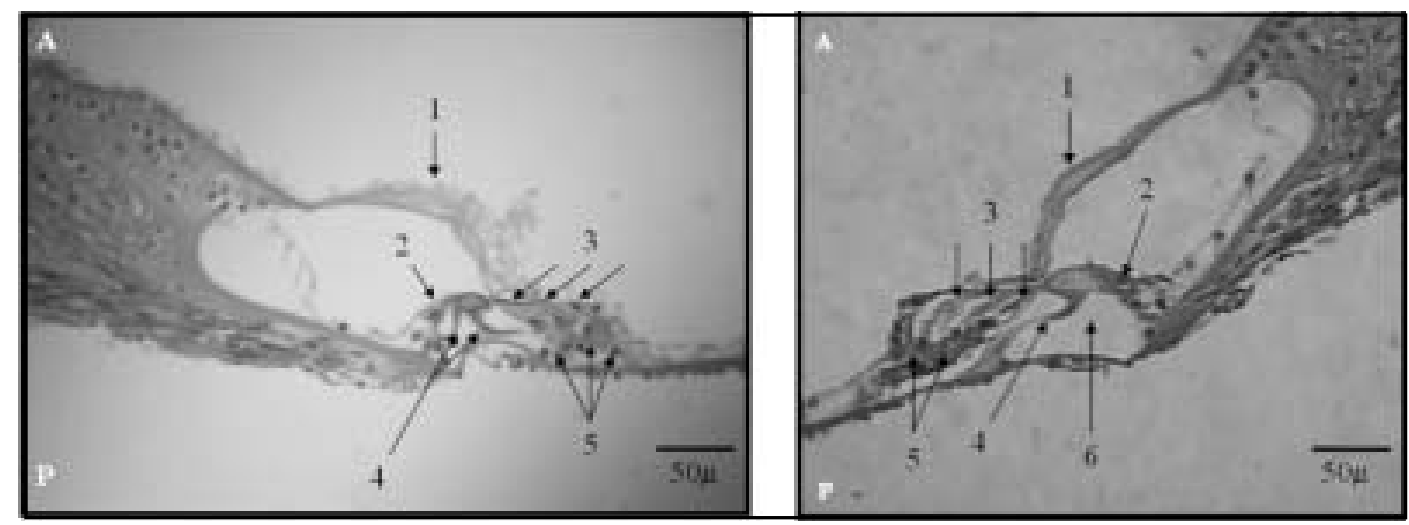

Figura 10. Dos microfotografías del órgano de Corti de chinchilla. 1. Membrana tectoria; 2. Célula ciliada interna; 3. Células ciliadas externas; 4. Células pilares; 5 . Células de sostén; 6 . Túnel de Corti.

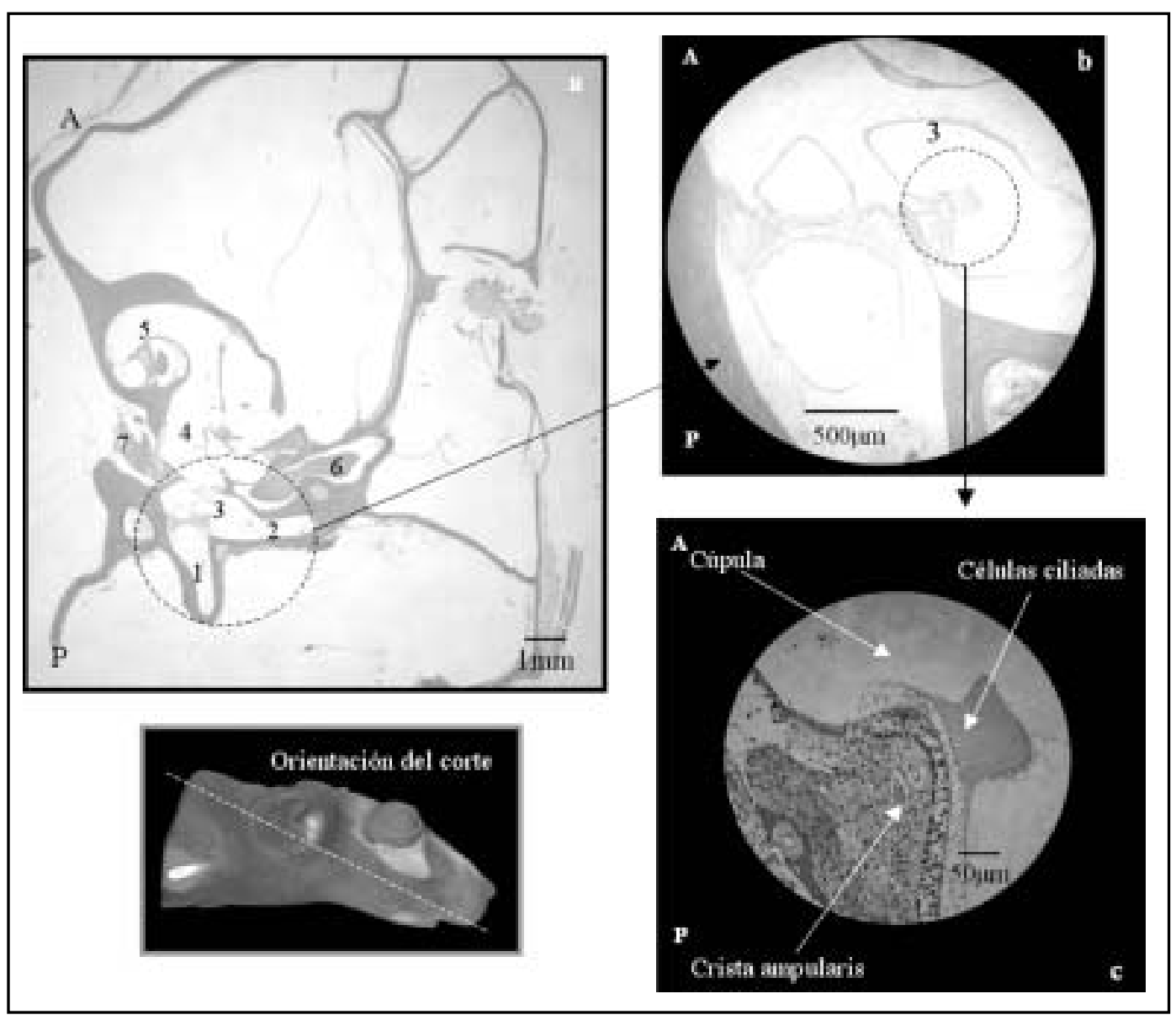

Figura 11. Microscopía de conductos semicirculares de chinchilla. En a se observa parte del oído interno: 1. Conducto semicircular posterior; 2. Conducto semicircular lateral; 3. Ampolla del conducto semicircular lateral; 4. Vestíbulo; 5. Cóclea; 6. Nervio facial; 7. Conducto auditivo interno. En b y c se observa un corte con mayor aumento, específicamente la cresta ampular del conducto semicircular lateral de chinchilla. A. Anterior; P. Posterior. 


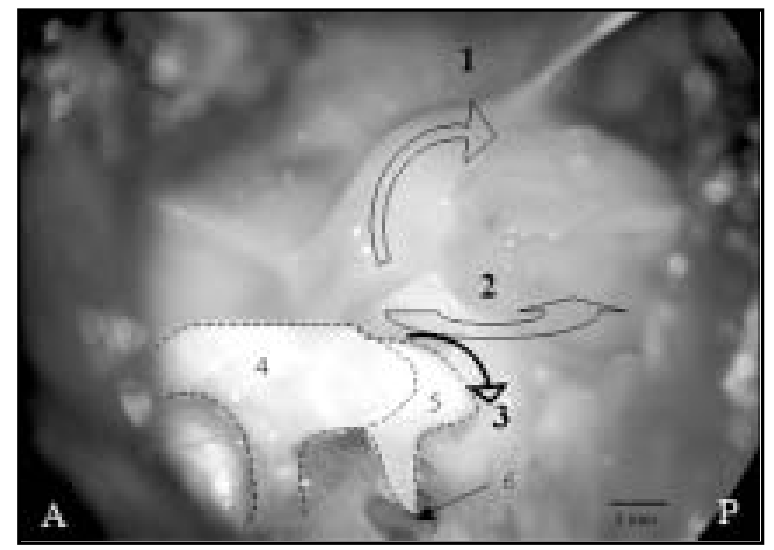

Figura 12. Pared posteromedial de cavidad timpánica de chinchilla. 1. conducto semicircular anterior; 2. Conducto semicircular lateral; 3. Canal del nervio facial; 4. Martillo; 5 . Yunque; 6 . Ventana redonda.

A diferencia del humano, en la chinchilla y el cobayo, el martillo y el yunque están unidos por cartílago (fusionados), formando sus cuerpos una barra horizontal ${ }^{7,8}$. Una de las hipótesis al respecto se orienta a la inercia de los osículos. Dado que la inercia de un sistema depende, entre otras cosas, de la masa, organismos grandes como el humano requieren mantener una articulación en bisagra entre el martillo y el yunque para disminuir su inercia. Sin embargo, en animales pequeños como la chinchilla, la masa de estos osículos es menor (disminuyendo también su inercia), y por eso se fusionan (no requieren una articulación en bisagra $)^{8}$.

En la chinchilla se observa entre las cruras el vestigio de la arteria estapedial, mientras que en los humanos normalmente no se presenta esta estructura ya que involuciona. Sin embargo se encuentra una arteria estapedial persistente en un $0,02 \%$ a $0,05 \%$ de la población ${ }^{9,10}$.

b. Oído interno

Cóclea: Es sabido que la cóclea tiene entre 1,5 a 4,5 vueltas sobre el modiolo dependiendo de la especie de mamífero ${ }^{11,12}$.En el cobayo, al igual que la chinchilla, da 31/2 vueltas, y su estructura ósea es fina, característica que se observa en varios roedores ${ }^{13,14}$. El hecho de que en la chinchilla, la cóclea presente una vuelta más en relación al modiolo, (con respecto a la del humano), le permite tener un amplio rango de frecuencias audibles.
Vestíbulo: Los conductos semicirculares de la chinchilla se identifican fácilmente al acceder al oído medio, dado que son proyectados hacia la gran bula. Están cubiertos por un delgado tejido óseo y su inervación está dada por el nervio vestibular? ${ }^{7}$. La sensibilidad de los conductos semicirculares se ha relacionado con el radio de curvatura de ellos ${ }^{15}$. De acuerdo a los datos reportados por Hullar, el conducto anterior de la chinchilla (por presentar un mayor radio de curvatura), tendría más sensibilidad que el conducto lateral y que el posterior. Además, demostró que los conductos semicirculares de la chinchilla, a pesar de ser no ortogonales, presentan sensibilidades similares en rotaciones en todas las direcciones ${ }^{16}$. Esto no ocurre en otros mamíferos pequeños, tales como el cobayo y conejo, ya que en ellos se observa una mayor sensibilidad al movimiento de rotación de la cabeza en una dirección y menor sensibilidad en otras ${ }^{17,18}$.

c. Consideraciones técnicas de la histología de la cóclea

El estudio histológico del hueso temporal y específicamente del órgano coclear es laborioso. Schuknecht define como artefacto a los cambios producidos durante la disección y preparación del tejido, y los clasifica en primarios y secundarios ${ }^{19}$. Los primarios son aquellos cambios que se esperan encontrar tras la preparación del tejido y que por lo tanto se consideran dentro del aspecto histológico 
"normal". Los artefactos secundarios son aquellos que son "anormales" para la técnica utilizada y que son resultado de fallas en la disección o en la técnica histológica ${ }^{19}$. Por este motivo se debe ser cuidadoso en cada paso, ya que las estructuras pueden ir alterándose en cada una de las etapas del procesamiento: antes de la fijación si ésta tarda mucho; durante el proceso de descalcificación, si éste se prolonga; y durante la inclusión y el corte. Durante la inclusión, hay que preocuparse de que la parafina llegue a todo el tejido, sobretodo si presenta cavidades aireadas, como es el caso de las bulas de la chinchilla. Con respecto al corte, influye la técnica y los materiales, como la navaja a utilizar, ya que si esto es inadecuado, los cortes resultarán con artefactos secundarios que impedirá una descripción adecuada.

\section{CONCLUSIONES}

La Chinchilla laniger, como modelo de investigación en audición, resulta extremadamente interesante ya que tiene estructuras similares al oído humano, tales como el estribo, órgano coclear, distribución de células ciliadas y sistema vestibular. Sin embargo, Ilama la atención diferencias que presenta con nuestro oído, como por ejemplo, la fusión del martillo con el yunque, la cual también se encuentra en otros animales pequeños como el cobayo.

El registro fotográfico obtenido (tanto macroscópico como microscópico) podrá ser utilizado como guía anatómica para los investigadores que trabajen en audición utilizando este modelo.

Tras realizar este trabajo, se abre la posibilidad de continuar estudiando el oído de este animal, optimizando cada vez más la técnica histológica del órgano y utilizando metodología más avanzada como por ejemplo una resonancia nuclear magnética, la cual permitiría definir con mayor precisión las partes blandas.

\section{BIBLIOGRAFÍA}

1. Spotorno A, Zuleta C, Valladares J, Deane A, JIMÉnEZ J. Chinchilla laniger. Mammalian Species 2004; 758: 1-9.
2. Grau J. La chinchilla, su crianza en todos los climas. 4a ed. Buenos Aires. Ateneo 1992.

3. Rasmussen GL. Efferent fibers of the cochlear nerve and cochlear nucleus. En: Rasmussen G.L, Windle W. Neural mechanism of the auditory and vestibular systems. Thomas, Springfield, III 1960; 105-15.

4. MILLER JD. Audibility curve of the chinchilla. $J$ Acoust Soc Am 1963; 35: 1907.

5. Cummings C, Haughey B, Thomas J, Harker L, Flint P. Cummings otolaryngology: Head and neck surgery. 4a ed. Mosby 1998; 149: 3373-87.

6. Heffner RS, Heffner HE. Behavioral hearing range of the chinchilla. Hear Res 1991; 52(1): 13-6.

7. Browning G, Granich M. Surgical anatomy of the temporal bone in the chinchilla. Ann Ool 1978; 87: 875-82.

8. Puria S, Sim JH, Shin M, Steele C. A gear in the middle ear. Stanford University. Sitio web: www.stanford.edu/ puria1/Conferences/ PuriaAR02007.pdf .

9. Moreano EH, Paparella MM, Zelterman D, Goycoolea MV. Prevalence of the facial canal deshicence and of persistent stapedial artery in the human middle ear: a report of 1000 temporal bones. Laryngoscope 1994; 104: 309-20.

10. StefFen TN. Vascular anomalies of the middle ear. Laryngoscope 1968; 78: 171-97.

11. Thorne M, Salt an, DeMott Je, Henson MM, Henson OW Jr, Gewalt SL. Cochlear fluid space dimensions for six species derived from reconstructions of three-dimensional magnetic resonance images. Laryngoscope 1999; 109 (10): 1661-8.

12. WYsockI J. Dimensions of the vestibular and tympanic scalae of the cochlea in selected mammals. Hear Res 2001; 161(1-2): 1-9.

13. WYsockı J. Topographical anatomy of the guinea pig temporal bone. Hear Res 2005; 199 (12): $103-10$.

14. Goksu N, Haziroglu R, Kemaloglu Y, Karademir N, Bayramoglu I, AkyiLdiz N. Anatomy of the guinea pig temporal bone. Ann Otol Rhinol Laryngol 1992; 101(8): 699-704.

15. Rabitt RD, Damiano ER, Grant JW. Biomechanics of the semicircular canals and otolith organs. En: Highstein S, Fay RR y 
Popper AN eds, The vestibular system. New York: Springer, 2004; 153-201.

16. Hullar T.E, Williams C.D. Geometry of the semicircular canals of the chinchilla (Chinchilla laniger). Hear Res 2006; 213: 17-24.

17. Curthoys IS, Curthoys EJ, Blanks RH, Markham $\mathrm{CH}$. The orientation of the semicircular canals in the guinea pig. Acta Otolaryngol 1975; 80 (34): 197-205.

18. Mazza D, Winterson BJ. Semicircular canal orientation in the adult resting rabbit. Acta Colaryngol 1984; 98(5-6): 472-80.

19. SCHUKNECHT HF. Pathology of the ear. $2^{\text {a }}$ ed. Philadelphia: Lea \& Febiger, 1993; 7-15.

* Esta publicación es parte del proyecto "Interferencia de la expresión de genes en el oído interno como herramienta molecular para regenerar células ciliadas" financiado por el concurso anual 2006 de la Sociedad Chilena de Otorrinolaringología, Medicina, Cirugía de Cabeza y Cuello, beca CONICYT doctorado y AT-24071080.

Dirección: Dra. Loreto Carrasco M. Las Violetas 2336 depto. 605

Providencia, Santiago, Chile. Teléfono: 7164663

E mail: loretocarrascomujica@yahoo.es 